\title{
Examination and Comparison of Theta Band Connectivity in Left- and Right-Hand Dominant Individuals throughout a Motor Skill Acquisition
}

\author{
Jessica McDonnell ${ }^{1, *}$, Nicholas P Murray ${ }^{2}$, Sungwoo Ahn ${ }^{3} \mathbb{D}$, Stefan Clemens ${ }^{4}\left(\mathbb{D}\right.$, Erik Everhart ${ }^{5}(\mathbb{D})$ \\ and J. Chris Mizelle 2 (D) \\ 1 Department of Neurological Surgery, University of Louisville, Louisville, KY 40202, USA \\ 2 Department of Kinesiology, East Carolina University, Greenville, NC 27858, USA; \\ murrayni@ecu.edu (N.P.M.); mizellej15@ecu.edu (J.C.M.) \\ 3 Department of Mathematics, East Carolina University, Greenville, NC 27858, USA; ahns15@ecu.edu \\ 4 Department of Physiology, East Carolina University, Greenville, NC 27858, USA; clemenss@ecu.edu \\ 5 Department of Psychology, East Carolina University, Greenville, NC 27858, USA; everhartd@ecu.edu \\ * Correspondence: jessica.mcdonnell@louisville.edu
}

Citation: McDonnell, J.; Murray, N.P; Ahn, S.; Clemens, S.; Everhart, E.;

Mizelle, J.C. Examination and

Comparison of Theta Band Connectivity in Left- and Right-Hand Dominant Individuals throughout a Motor Skill Acquisition. Symmetry 2021, 13, 728. https://doi.org/10.3390/sym13040728

Academic Editor: Luca Paolo Ardigo

Received: 22 February 2021

Accepted: 14 April 2021

Published: 20 April 2021

Publisher's Note: MDPI stays neutral with regard to jurisdictional claims in published maps and institutional affiliations.

Copyright: (c) 2021 by the authors. Licensee MDPI, Basel, Switzerland. This article is an open access article distributed under the terms and conditions of the Creative Commons Attribution (CC BY) license (https:// creativecommons.org/licenses/by/ $4.0 /)$.

\begin{abstract}
The majority of the population identifies as right-hand dominant, with a minority $10.6 \%$ identifying as left-hand dominant. Social factors may partially skew the distribution, but it remains that left-hand dominant individuals make up approximately 40 million people in the United States alone and yet, remain underrepresented in the motor control literature. Recent research has revealed behavioral and neurological differences between populations, therein overturning assumptions of a simple hemispheric flip in motor-related activations. The present work showed differentially adaptable motor programs between populations and found fundamental differences in methods of skill acquisition highlighting underlying neural strategies unique to each population. Difference maps and descriptive metrics of coherent activation patterns showed differences in how theta oscillations were utilized. The right-hand group relied on occipital parietal lobe connectivity for visual information integration necessary to inform the motor task, while the left-hand group relied on a more frontal lobe localized cognitive based approach. The findings provide insight into potential alternative methods of information integration and emphasize the importance for inclusion of the lefthand dominant population in the growing conceptualization of the brain promoting the generation of a more complete, stable, and accurate understanding of our complex biology.
\end{abstract}

Keywords: theta oscillations; imaginary coherence; hand dominance; skill acquisition; graph theory

\section{Introduction}

Functional roles of distributed brain regions connected by dense axonal networks are defined by their inputs and outputs [1,2]. The complex circuitry connecting brain regions is a critically important aspect of brain function, enabling the coordination of distinctly different brain areas. Brain functions support adaptable and complex movements. The expression or suppression of movement is weighted by cognitive and sensory processes that can be mathematically simplified and represented as a Bayesian inference, an outcome weighted by two sources of information: the current sensory state and memories of previous sensory states. Theoretical neuroscientists often include this Bayesian decision theory to conceptualize an internal model that governs motor behavior and have shown these mechanisms to be critical for learning new movements and skills [3-6]. The continuous and coordinated integration of sensory information, both about the environment and the current state of the body, is presumably used to determine the appropriate set of muscle forces needed to generate a desired movement or action. The coordination between the nervous system and the musculoskeletal system enables the sensorimotor 
plasticity that fosters adaptability. Sensorimotor integration is a multifaceted fusion of incoming signals condensed into one outgoing motor message. One of the factors to influence sensorimotor integration properties and processes is hand dominance. Prior work has shown that left- and right-hand dominant individuals differ in their structural and functional neural organization, overturning previously held assumptions of hemispheric chirality [7]. Hand dominance is now understood to influence fundamental interactions with the environment [8].

Functionally, hand dominance indicates an innate asymmetrical preference for the use of one hand over the other. Structurally, hand dominance was associated with a comparatively larger volume of the hand motor cortical area contralateral to the dominant hand, however recent findings have found the changes in brain structure associated with left handedness to be more subtle [9]. The matter, which is no longer conclusive, highlights again, the lack of understanding regarding hand dominance preference. Behaviorally, left-hand dominant (LH) individuals tend to present as more bilateral compared to right-hand dominant (RH) individuals [10-12]. Thus, neurologically, hand dominance results in organizational tendencies influencing the networks that form the communication pathways necessary for the signal transfer responsible for facilitating all complex thought and behavior, including the acquisition and regulation of movement. A study examining twins with different limb dominance found that bilateral occipital and frontal resting state electroencephalographic (EEG) spectral power was a covariate of hand dominance [13]. Another study with left- and right-hand dominant participants observing images of left and right hands executing a motor task found clear differences in neural activation patterns [14]. In this study by Kelly et al., RH individuals observing right- and left-handed task execution exhibited connectivity patterns distinctly lateralized to the hemisphere contralateral to the hand observed, whereas LH observers presented with a more bilateral distribution of neural connectivity regardless of the hand observed [14]. Evidence now supports the hypothesis of underlying difference in the neural networks of left- as compared to righthand dominant individuals. However, the exact nature and extent of how the populations differ, and especially how hand dominance influences, or is influenced by, neurological organization in the context of overt motor control and sensorimotor integration, is largely unknown and unreported in the literature. This is in part due to the exclusion of the left-hand dominant populations in motor control studies [15].

The arrangement of cortical networks enabling signal transfer and the propagation of information is an area of active interest in neuroscience research. The nervous system enables adaptation through neural coordination, organization, and neuromodulation. Anatomical connections between brain areas provide the structural framework enabling all conscious thought and behavior. Mapping these neural networks to understand brain function can provide information related to interregional information processing. The current work was designed to examine the nature of the underlying functional, neurobiological differences driving limb preference in the context of motor performance. A dynamic, visually-based force matching task involving dominant and non-dominant hands was selected due to previous findings showing the two populations to have behavioral differences [16]. Visual feedback was provided in real-time as movement errors in the visual domain have been shown to influence cortical motor areas and shape neural activity in motor and premotor regions [17-20]. Continuous EEG was used to capture coherent neurological activations, allowing for the analysis of network activation patterns known to undergo reconfiguration on multiple time scales, thus enabling observation of how communication patterns change and develop with task repetition. The signal was processed to enable the analysis of functional connectivity, a measure related to the temporally dependent activation of distinct brain regions, representing transient relationships between distinct neuronal populations. Cortical regions dynamically couple to form functional networks that can be modeled as a set of mathematical dependencies between activations of discrete neuronal populations or regions. Information encoded within oscillation patterns serves to unite assemblies of neurons in either a state of activation or inhibition 
and can be measured from the EEG signal [21]. Each oscillatory cycle (wavelength) is a temporal processing window, indicative of the initiation and termination of an encoded message [21,22]. Generally, slower oscillations with longer cycle lengths (periods) can transfer information to more remote locations, and often serve as global integrators. The purpose of this study was to analyze the slow-wave theta band activity due to the novelty of the task combined with practice and skill acquisition. Graph theory was applied to represent the whole brain as a system and quantify interacting elements. In the context of EEG, electrodes and underlying brain regions are defined as nodes with the edges as measures of statistical association between the nodes. Graph Theory metrics, degree, clustering coefficient, and global efficiency, were calculated to provide a description of the network properties assigned to populations of hand dominance.

Based on the experimental design and the current state of the literature, our central hypothesis was that the two hand dominant populations ( $\mathrm{LH}$ and $\mathrm{RH}$ ) would present with different neural activation patterns. Specifically, we expected a more bilateral network dispersion, with greater cross hemisphere communication for LH using both dominant and non-dominant hands. We expected this bilateral activation to be reflected by a comparatively greater global efficiency. Conversely it was hypothesized that RH would have more lateralized patterns expressed along the dominant hemisphere and decreased global efficiency. We further expected that theta band connectivity would decrease with the progression of trials, denoted by a diminished degree, for both groups. Finally, in the nondominant conditions, we expected the LH group would show a higher clustering coefficient reflecting a more stable strategy as compared to the RH group using the non-dominant left hand. What we found, was the two groups encoded the task differently, captured in the differing patterns of theta connectivity. The different strategies imply different uses of theta oscillations. Hand dominant groups were found to potentially optimize different aspects of the task influencing tactics of skill acquisition.

\section{Materials and Methods}

\subsection{Subjects and Experimental Design}

Twelve left-hand dominant (9 female 3 male) and twelve right-hand dominant ( 4 female 8 male) individuals aged 18-35 participated in the study (Figure 1). Handedness was determined by the Edinburgh Handedness Inventory [23], and potential participants with a handedness score between -40 and +40 were considered ambidextrous and excluded from the study. All participants self-reported as healthy with no previous neurological or musculoskeletal injuries affecting upper extremity motor function, and all had normal or corrected-to-normal vision. Experimental procedures were approved by the University and Medical Center Institutional Review Board at East Carolina University, and informed consent was obtained from all participants before participation. The inequitable distribution of genders across the groups was not initially controlled for and do present a potential limitation in the interpretation of the results. Gendered effects for hemispheric asymmetries have been reported, however these findings between genders are subtle, only demonstratable with much large sample sizes and are not directly related to the present task [24-26].

EEG data were collected during three conditions of a visually guided, handgrip force modulation task. A $5^{\prime} \times 3^{\prime}$ monitor displayed a target moving linearly along a repeating trajectory (Figure 2). Subjects were instructed to use hand force scales (Innovative Sports Training, Chicago, IL, USA) to control a cursor, also displayed in real-time, with the goal of keeping the cursor within the target. Magnitude of force output correlated with the distance from the origin; as the target moved further away from the origin, more force was required to keep the cursor within the target. Target forces fell between the range of 0-6.25 N. Each force trajectory pattern duration was approximately twenty-two seconds, with the target moving at a pseudo constant rate of $1.6 \mathrm{~cm} / \mathrm{s}$ to ensure that the participants were vigilant in completing the task. This was accomplished with occasional points of mandatory contact between target and cursor, if contact was not made, the target would pause until contact was made and then it would continue along its trajectory. Participants completed ten 
consecutive trials with their dominant hand, ten consecutive trials with their non-dominant hand, followed by ten consecutive trials with both hands simultaneously. The bimanual condition followed the same pattern as both unimanual conditions with a single cursor traversing between the two axes (horizontal and vertical) with each hand controlling the direction practiced in the unimanual conditions. Between each trial, a six-second rest period was sampled to collect baseline data. A minimum two-minute break occurred between dominant, non-dominant, and bimanual conditions. The non-randomized order of conditions was an effort to best mimic general strategies for learning; dividing a complex task into simpler subtasks to be learned independently [27]. The dominant unimanual condition served as a period for structural learning in which subjects explore how to maximize information and assign errors. Maintaining the structure of the task applies the skills acquired in structural learning to initiate parametric learning. This technique has been used as a method to "speed up learning" [28-31].

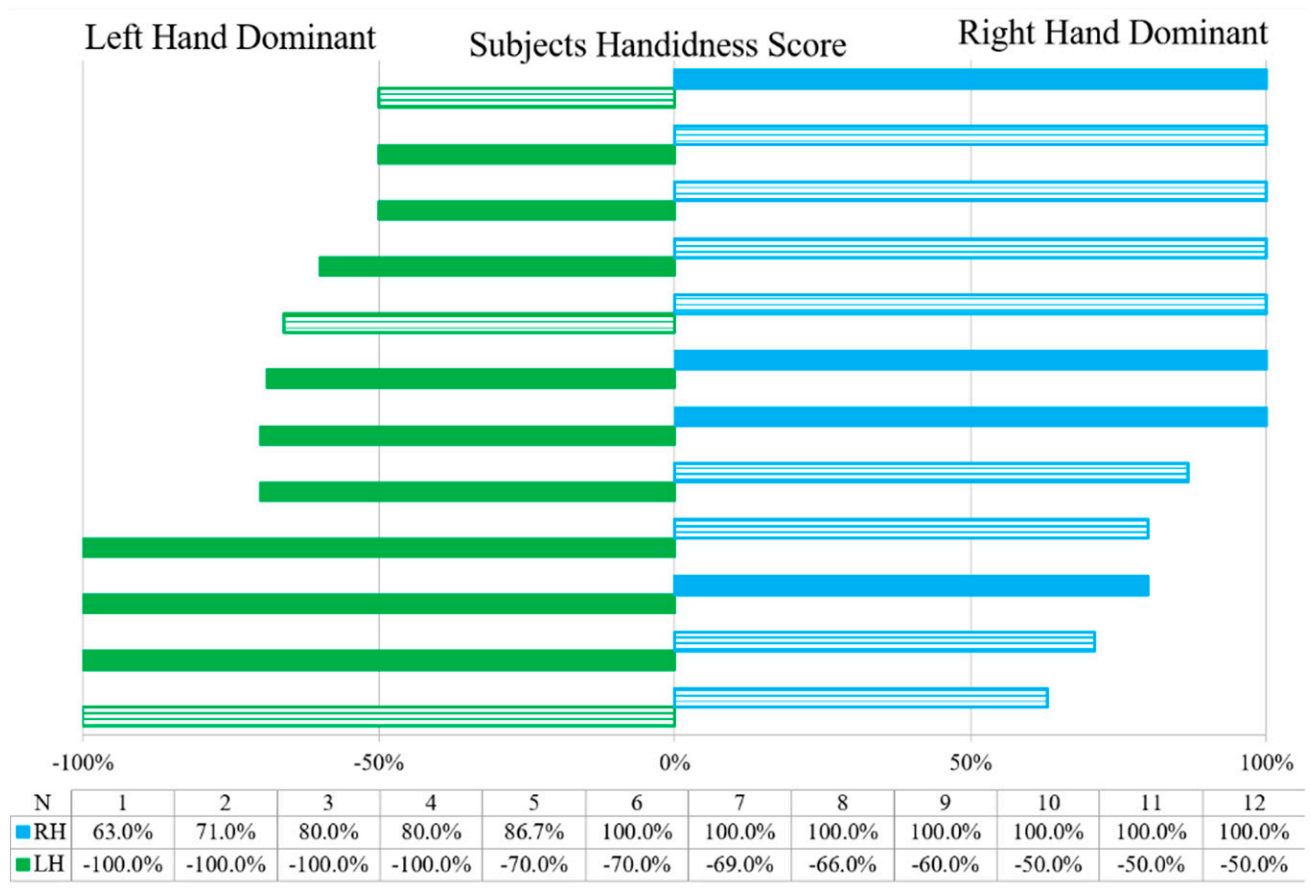

Figure 1. Handedness scores for left and right participants with females denoted with a solid bar, males with stripes.

\subsection{Data Analysis}

EEG data were collected at $1 \mathrm{kHz}$ using a 64-channel cap (Compumedics Neuroscan, Charlotte, NC, USA) placed on the scalp in accordance with the international 10-20 system, with impedance kept below $10 \mathrm{k} \Omega$. Throughout the recording, events were marked using an outgoing voltage pulse that was triggered with a conditional force and target threshold (if $<3 \mathrm{~V} \&$ target $\neq 0$ ), marking the beginning and end of each trial. Continuous EEG data were exported from the acquisition software (CURRY 7; Compumedics Neuroscan, Charlotte, NC, USA), preprocessed with functions from the EEGLAB toolbox [32], and then analyzed with custom Matlab software (The Mathworks, Natick, MA, USA). Data were cleaned using high [1 Hz] and low [55 Hz] pass filters, linear detrended, and then referenced to the average of the montage. Artifact subspace reconstruction [33] was used for the removal of ocular and muscular artifacts. A Laplacian transform was applied as a spatial filter to remove the potential for volume conduction artifact [34]. Data were downsampled to $250 \mathrm{~Hz}$ for subsequent analysis. A time-frequency wavelet decomposition was performed on each trial of $21.875 \mathrm{~s}$. with a 3-12 cycle range and 1 to $40 \mathrm{~Hz}$ minimum and maximum frequency range. The complex cross-spectrum was calculated for each of the 62 channels yielding a symmetrical matrix describing the correlation between all electrodes at each 
time point, and each frequency from 1 through $40 \mathrm{~Hz}$. The first three trials (1:3) for each condition (dominant, non-dominant, bimanual) and group (left hand dominant [LH] and right hand dominant $[\mathrm{RH}]$ ) were averaged to represent the early phase, while the final three trials $(8: 10)$ were averaged to represent the late phase, the middle trials were not included in the analysis. The real and imaginary coherence between all channel pairs were calculated from the complex cross-spectrum for each of the trial bins (early, late, and baseline) and conditions (unimanual dominant, non-dominant, and bimanual) [35]. A corrective factor was applied to the imaginary coherence to account for artificial suppression of connectivity values near true sources [36]. The absolute value of the corrected imaginary coherence was taken and theta $[4-7 \mathrm{~Hz}]$ was extracted from the signal and normalized against the baseline data, for frequency-specific normalization.

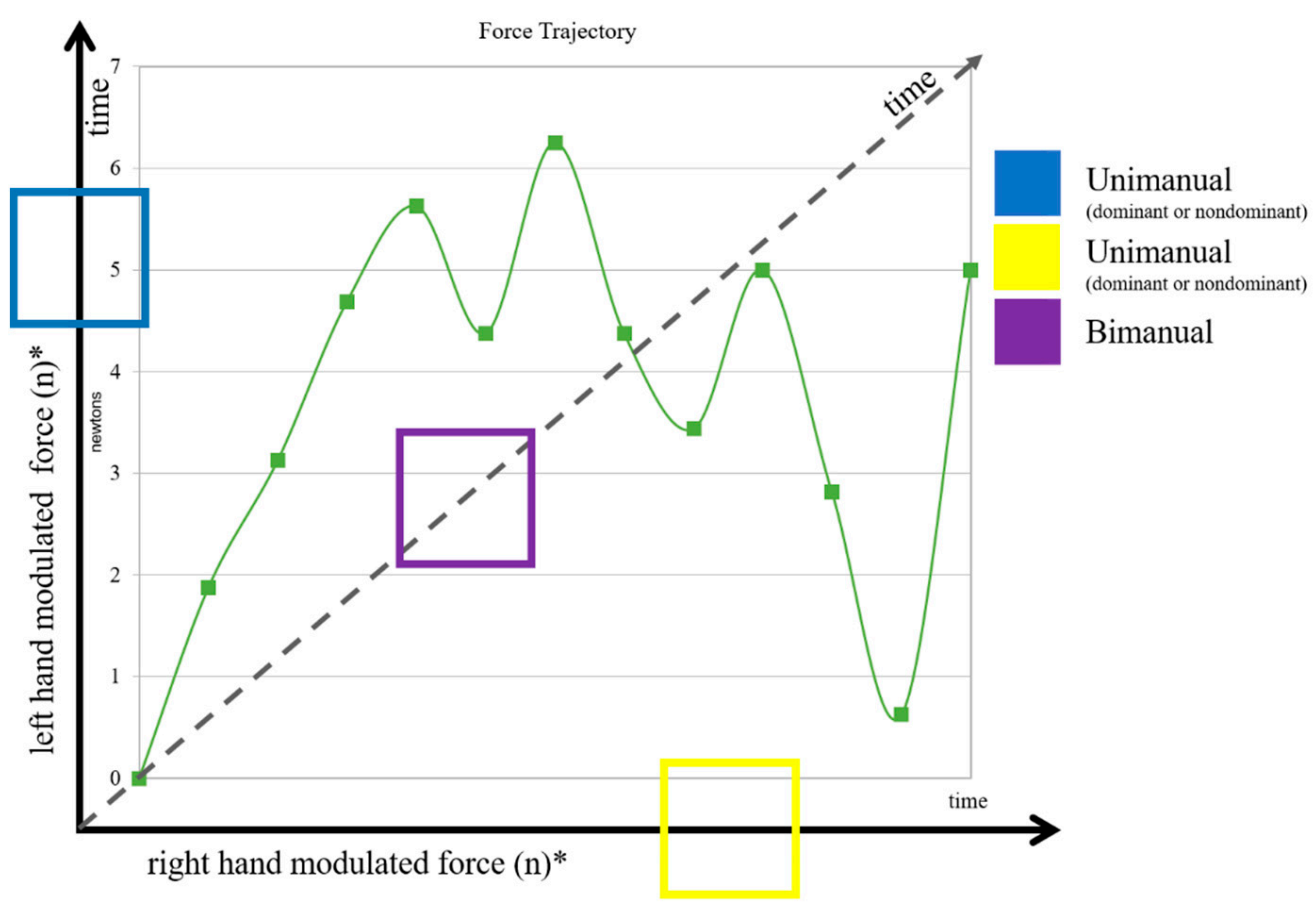

Figure 2. Target force trajectory displayed in green with the points of mandatory cursor contact marked with squares. Conditional directions shown with boxes, unimanual in yellow and blue, bimanual in purple; denoting the target path and force modulated direction of the cursor. Asterisk on the $\mathrm{x}$ and $\mathrm{y}$ axis indicate an example set up, hand axes combo was split within groups such that half of each LH and RH group used dominant hand for each horizontal and vertical direction.

\subsection{Graph Theory Metrics}

In an effort to understand the brain as a dynamic system, the whole brain was taken into consideration and evaluated as a network. We employed a branch of mathematics, graph theory, which focuses on the properties and behaviors of networks defined as systems consisting of a set of nodes (electrodes) linked by edges (connections or interactions) [37]. As described above, we first determined connectivity profiles associated with the different groups ( $\mathrm{LH}$ and $\mathrm{RH}$ ) and conditions to generate a profile of statistical dependency between brain regions (nodes). This enabled the evaluation of how network elements interacted [38]. From these networks, we calculated several graph theory measurements. Degree, a node parameter indicative of the number of links connected to a particular node, suggests a level of interaction for that node with other nodes in the network. This allowed for indirect quantification of network, or signal, density [39]. Degree was calculated by determining how many connections each of the electrodes made with each other. Hubs were defined as nodes possessing a degree of four or greater. The clustering coefficient metric determines the local interconnectedness and has been associated with high local efficiency of information transfer and stability [40]. The clustering coefficient was calculated as the faction of closed 
triangles among three nodes. Contrarily, global efficiency was established to determine the connectedness of spatially distant regions that are necessary for the integration of specialized information but may be costly in terms of energy and material [41]. Global efficiency was computed by finding the distance between each of the connected nodes. For each of the metrics, an average was taken for each of the groups per conditions and time. Graph metrics are all depicted and reported as a way of quantifying the imaginary coherence networks for each of the groups (figures located in Appendix A).

\subsection{Statistics}

No assumption could have been made about the underlying distribution of the data, thus a nonparametric permutation statistical approach, based on the FieldTrip toolbox [42], was taken. At the individual participant level, corrected imaginary coherence data were used to create a null statistical distribution or a distribution that would be true if there was no dependence on specific channel pairs in the actual distribution of connectivity estimates. This was accomplished by randomly permuting electrode labels through 1000 permutations. A Fisher's Z-statistic map (Zmap) was then calculated. A critical value $(\mathrm{t}=1.6449$ for $p<0.05$ ) was then used to threshold the Zmap, therein removing values falling below the critical value. The Zmap was then used to mask the true connectivity matrix, leaving only connectivity values that were statistically reliable according to the permutation test. A similar statistical permutation process occurred at the group level for comparisons of interest. First, all thresholded individual adjacency matrices were made symmetrical and then concatenated for two conditions (i.e., LH and RH). The true connectivity difference was calculated as the difference of the means of the subject-specific connectivity matrices across conditions. The null distribution was then calculated as above, after both group and electrodes labels were shuffled through 1000 permutations. A similar process was used to calculate a Zmap of the condition differences, and the true difference matrix was thresholded to leave only connectivity values that were statistically reliable according to the permutation test. Each comparison yielded two difference matrices depending on the sign of the differences in the true difference matrix (e.g., Condition $\mathrm{A}>$ Condition B and Condition B $>$ Condition A). Post hoc t-tests were used to determine appropriate testing of the graph theory metrics. With significance $(p<0.05)$ a mixed design repeated measures ANOVA (factors and levels) was used to determine group, condition and time differences, with significance set at $p<0.05$. Effect size was calculated using Cohen's d and reported in the Results Section. Group variance was determined using standard error of the mean, $(\mathrm{SD} / \sqrt{ }(\mathrm{n}))$.

\section{Results}

Neurological findings are reported as differences maps with each group comparing the significant coherence patterns and reporting the significant differences found between the two conditions (Figure 3a). Importantly, the results discussed and displayed are not expressing raw activation, but rather the patterns of connectivity that are different across conditions. Weighted imaginary coherence maps depicting raw activation patterns for each group, condition, and time bin, are located in Appendix A. The overall purpose of the study was to identify patterns of cortical activation unique to left-hand dominant and right-hand dominant individuals evoked upon the repeated performance of the dynamic force regulation task with high levels of visuomotor integration. Graph network metrics were applied to quantify the differences. Differences between LH and RH groups executing the motor task are clear when looking within the theta frequency band. Importantly, differentially adaptable motor programs are seen to be detectable on a 10-trial time scale.

Group conditions were separately evaluated in two phases, or time bins: an initial phase (first three trials), and a final phase (last three trials) (Figure 3a). Generally, the RH group (bottom portion of Figure 3a) had comparatively greater reliance on posterior connectivity, suggesting a reliance on visual information to complete the task. The LH group [top portion of Figure 3a] had comparatively more frontal connectivity suggesting 
a more cognitive strategy that relied on executive function. The network metrics were calculated on each group's connectivity matrix (not the permuted differences maps) and are thus reflective of the actual activation patterns rather than descriptions of the permuted differences between the groups. All network metric findings are displayed however, only clustered coefficient and global efficiency were statistically allowed to be evaluated with a post hoc t-test. The significantly different global efficiency findings allude to fundamentally different neural strategies adopted by the two-hand dominant populations, a manifestation of a different utilization of slow-wave theta oscillations.

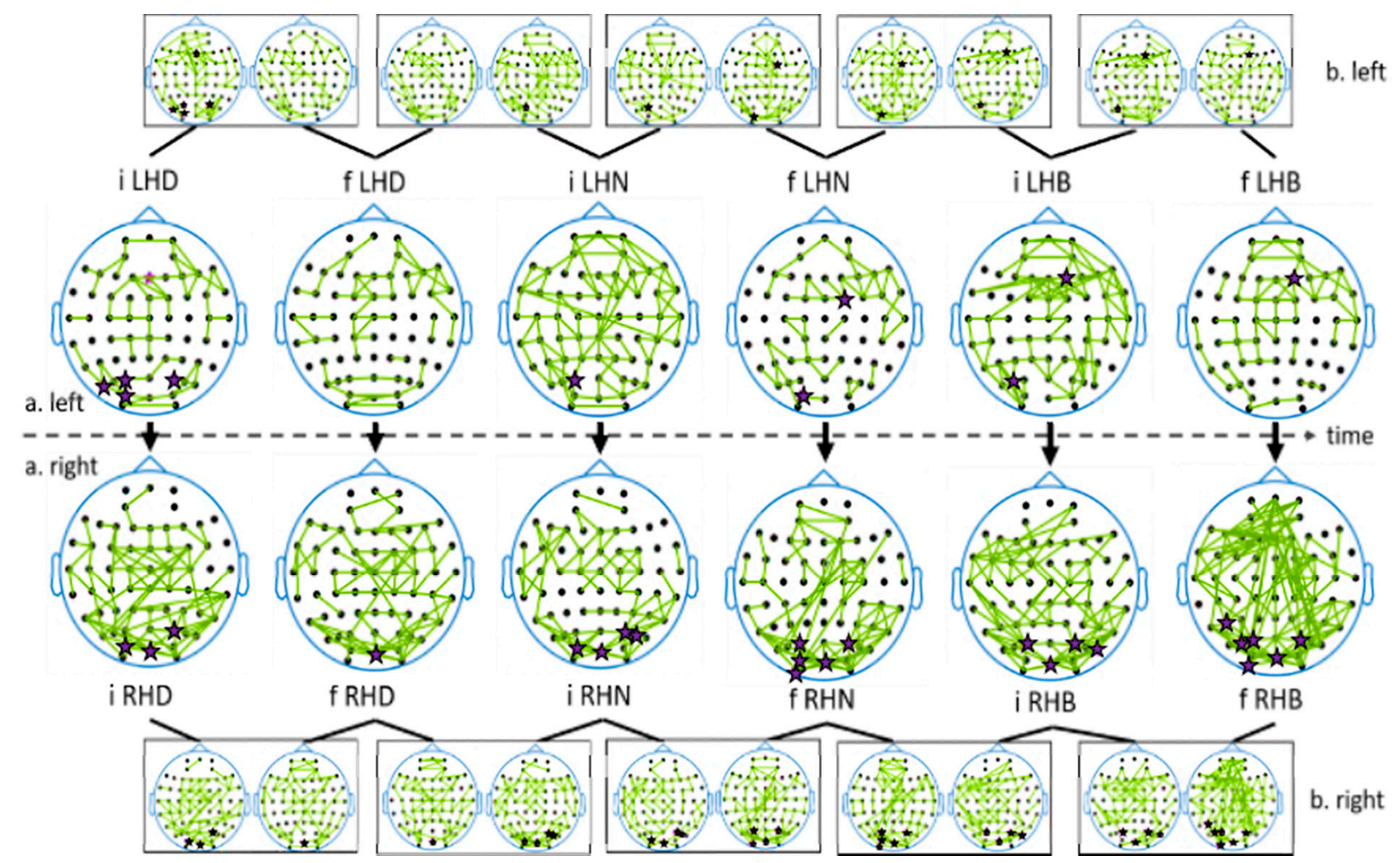

Figure 3. The large head plots labeled a. display differences across populations (LH-top RH-bottom) within the condition and trial bins (initial (i) final (f) dominant hand (D) non-dominant hand (N) bimanual (B)). The smaller head plots labeled $\mathbf{b}$. display coherence difference maps within groups as the experiment progresses across trials (initial to final) and conditions (unimanual dominant, unimanual non-dominant, bimanual). When present, hubs are denoted with a star over the corresponding electrode.

\subsection{Initial and Final-Dominant Hand (Condition 1)}

The initial three trials of the task were executed with the dominant hand. The RH group presented with differential bilateral connectivity, particularly along the posterior occipital and parietal-occipital regions, as well as over the midline motor area. The LH group had connectivity differences expressed anteriorly along the midline frontal area. Both groups possess occipital lobe hub locations while only the LH group had a frontal lobe hub. In the final dominant hand conditions, the RH group showed bilateral midline posterior, midline, and frontal lobe connectivity that extended laterally. The LH group showed a posterior shift in the frontal lobe connectivity, with an additional increase in LH temporal lobe connectivity. The increase in global efficiency in the RH group is suggestive of improved global integration. 


\subsection{Initial and Final-Non-Dominant Hand (Condition 2)}

The LH group using the non-dominant hand initially had a much more dispersed bilateral connectivity pattern ranging between anterior and posteriorly as compared to the $\mathrm{RH}$ groups using the non-dominant hand. The $\mathrm{RH}$ group appeared to adopt a very similar strategy when using their dominant hand (difference maps of the same hand across time are shown in Figure 3b), which is supported by the reemergence of hubs in the occipital lobe. Transitioning into the later trials, the RH group again showed occipital lobe connectivity extending anteriorly with a noteworthy right lateral parietal occipital network. The LH group's final non-dominant trials exhibited much more condensed connectivity differences with an anterior-posterior shift of connectivity, with another connectivity shift toward the right lateral hemisphere accompanied by a hub. A left lateral parietal occipital network also remained, again marked with a hub. The LH group had a decreased clustering coefficient between final dominant and non-dominant trials and increased global efficiency, which was indicative of a generally diminished need for strategy stability. Rather, the group adopted a stable neural activation strategy allotting for increase efficiency via global integration. Alternately, the RH group had an increase in the clustering coefficient and decreased global efficiency, suggestive of the need for signal stability rather than information integration.

\subsection{Initial and Final-Bimanual (Condition 3)}

The bimanual conditions did not present with fundamentally different connectivity patterns that were engaged during unimanual trials. The LH group had a midline frontal lobe pattern with lateral networks extending posteriorly. These networks diminished in the final trials with increased midline activation extending into the midline motor regions. The RH group again utilized bilateral occipital lobe connectivity, along with some posterior frontal lobe connectivity with a left hemisphere consolidation. Additionally, the RH groups had a marked increase in signal action particularly extending anteriorly from the occipital lobe and along the left lateral hemisphere frontal lobe to the midline. Here again, the network properties were shown to differ with the LH group showing an increase across network metrics while the RH group showed an increase in global efficiency only.

\subsection{Graph Network Metrics}

The graph network metrics of degree (Figure 4), clustering coefficient (Figure 5) and global efficiency (Figure 6) were modulated along with the coherent activity (Figure 3) as the two groups expressed different patterns across trials and conditions. Between the two groups, degree appeared to be diminished in the right-hand group compared to the left-hand group across initial (Cohen's $d=0.6591)$ and final $(d=0.8994)$ dominant hand conditions. The clustering coefficient was significantly decreased in the final bilateral condition from $\mathrm{LH}(0.06)$ to $\mathrm{RH}(0.04)(d=0.1109)$. The global efficiency was significantly different between groups in the initial dominant (LH: 0.14, RH: 0.12) $(d=0.5091)$, final non-dominant (LH: 0.19, RH: 0.1) $(d=1.0532)$, and initial bilateral (LH: 0.15, RH: 0.17) $(d=0.2540)$. The LH clustering coefficient decreased from final dominant to final nondominant hand conditions $(d=0.1109)$ while the trial progression from initial bimanual to final bimanual experienced a significant increase in the clustering coefficient $(r=0.1400)$. The global efficiency measure in the LH group was significantly increased with repetition from the final dominant hand to initial non-dominant $(d=0.4690)$ and then to both initial and final bimanual trials $(d=0.5569)$. This pattern was not seen to the same extent in the RH group, who showed a global efficiency increase in the dominant $(d=0.8973)$ and bimanual $(d=0.8419)$ hand conditions from initial to final trials but a decrease in the initial to final non-dominant condition $(d=0.5790)$. 


\section{Theta Degree}

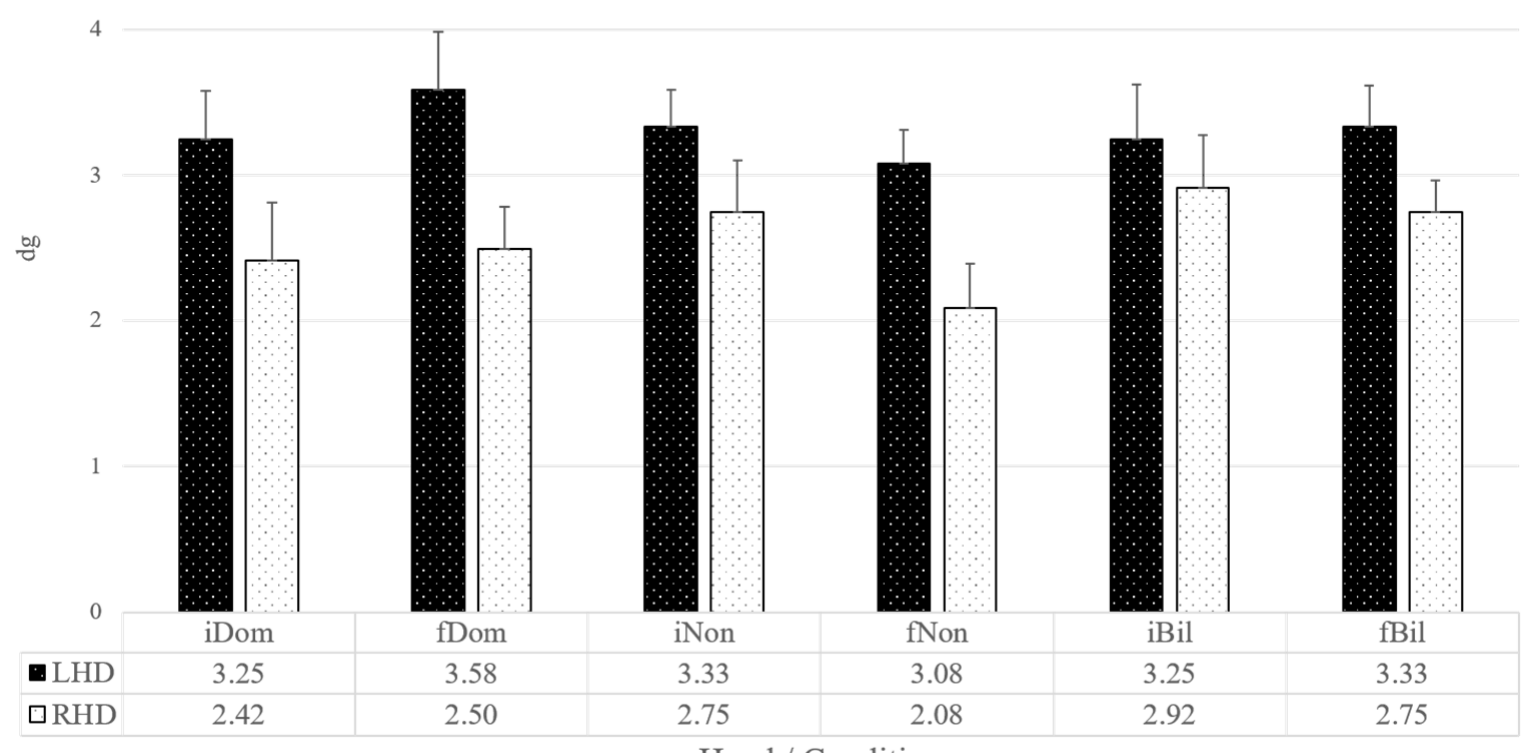

Hand / Condition

Figure 4. Left- and right-hand network metric degree across time and conditions with corresponding SD/ $\sqrt{ }(\mathrm{n}) \mathrm{displayed}$ with error bars. No post hoc testing was warranted for degree; however trends were not consistent between groups and are thus displayed.

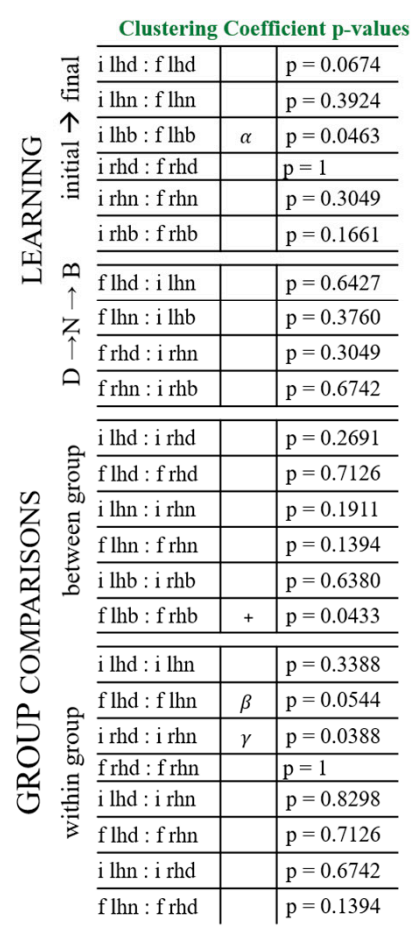

Theta Clustering Coefficient

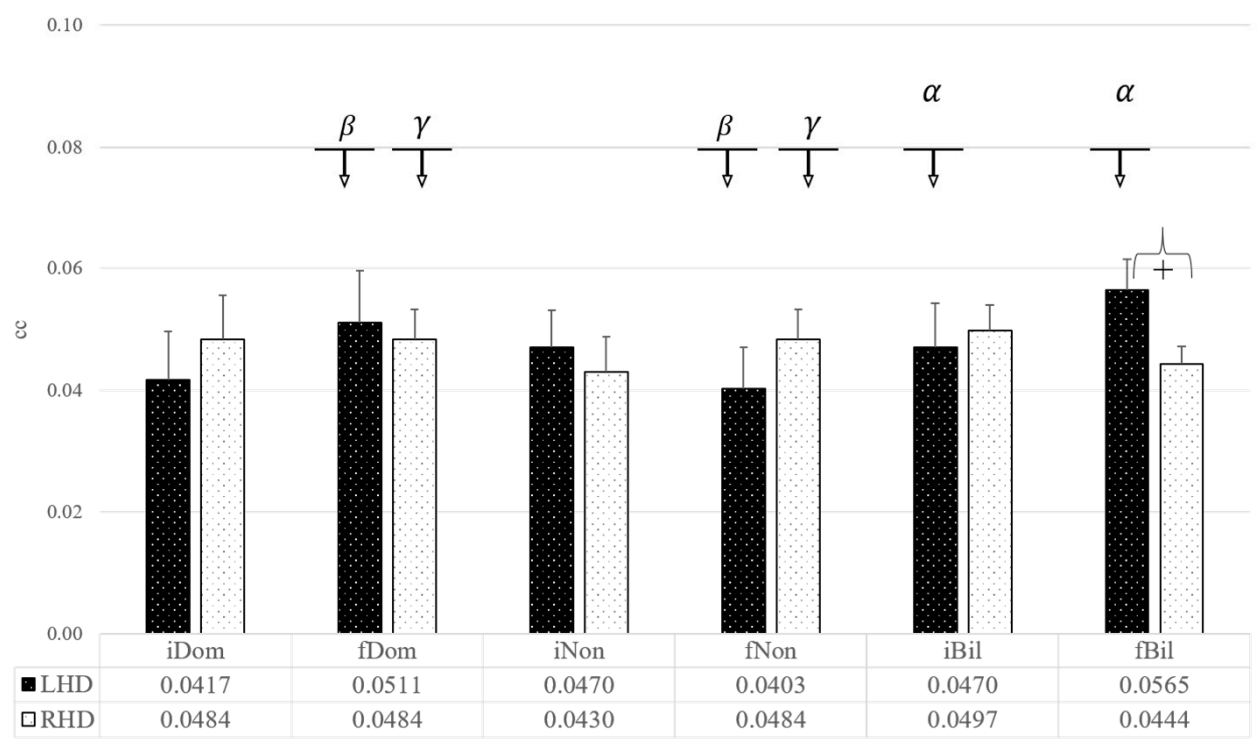

Hand / Condition

Figure 5. Left- and right-hand network metric clustering coefficient across time and conditions with corresponding SD $/ \sqrt{ }(\mathrm{n})$ displayed with error bars, group average values denoted in the table beneath. $p$ values denoted in the table to the left with significance between groups within a condition denoted with ' + ' across conditions significance are marked with matching Greek letters in the table and figure. 


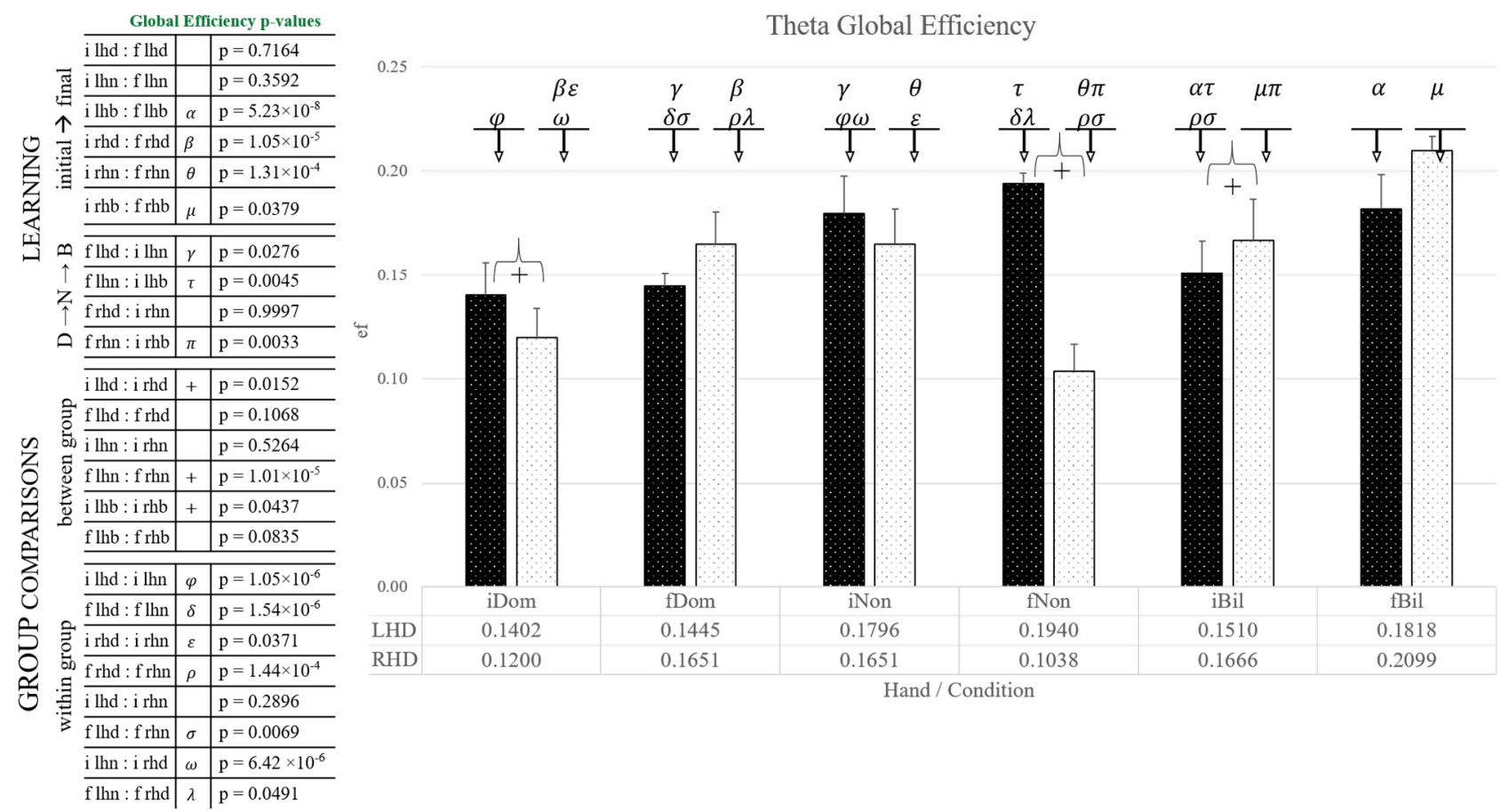

Figure 6. Left- and right-hand network metric global efficiency across time and conditions with corresponding SD/ $\sqrt{ }(\mathrm{n})$ displayed with error bars, group average values denoted in the table beneath. $p$ values denoted in the table to the left with significance between groups within a condition denoted with ' + ' across conditions significance are marked with matching Greek letters in the table and figure.

\section{Discussion}

In the present study, we examined cortical communication strategies in left- and right-hand dominant individuals and compared dynamic force tracking tasks guided by visual input. Subjects completed 10 trials for each of the three conditions, using first their dominant hand to modulate a cursor via dynamic force output, followed by their nondominant hand, and finally the bimanual modulation of a single cursor tracking the target moving along the resultant trajectory of the two unimanual conditions executed just prior, with each hand controlling the respective direction of the cursor assigned in the initial unimanual conditions. The organization of the trials was intended to promote learning [28]. The first three trials were averaged as an early representation of motor strategy, and the last three trials were averaged to represent the developed strategy after skill acquisition. Communication pathways were determined using corrected imaginary coherence, and results represent neural connectivity difference maps that highlight dissimilarities found between groups, trials, and conditions.

Previous work has shown that LH and RH individuals exhibit outward behavioral discrepancies and differences in connectivity patterns when observing actions [14]. Those neurological differences could be simplified into hemispheric dichotomies. However, we found that group differences in a dynamic motor execution task did not follow those from the action observation-based studies. We hypothesized that theta band connectivity would decrease with trial progression, which would be marked by a decreased degree. This was not found to be generalizable for either group, although the LH group did have diminished degree in both unimanual dominant hand conditions, due to the ANOVA, it did not qualify for post hoc testing, and thus no further extrapolation is possible. The greater cross hemispheric communication that we hypothesized would exist in the LH group was also not explicitly supported; the LH group presented with comparatively greater frontal theta midline activity and the RH group had comparatively greater bilateral occipital-parietal activity, thus the finding could not be generalized into hemispheric tendencies or patterns. The hypothesized greater global efficiency in the LH group was generally found to be 
supported; while not as simple as predicted, a statistically significant decrease across time was found within the LH group and not in the RH group. Our hypothesis of increased behavior of networks regarding degree and clustering coefficient was unfounded. While these hypotheses were generally not supported, the findings do suggest that the network metrics may be of value when evaluating skill acquisition. Our data suggest that initial strategies between LH and RH dominant groups differed, and furthermore, that as the trials progressed and cortical reorganization occurred, group strategies remained distinct.

The relatively slow rate of theta oscillations allows for long-range transfer of information, necessary for global integration. However, even the slow-wave theta can have rapid local influence necessary for error detection and correction [43-45]. As such, the role of theta oscillations has been partly determined by location $[44,46]$. The presence of frontal midline theta is reflective of cognitive control and error monitoring, while spatial and memory encoding occurs posteriorly at the parietal occipital junction [43-45]. Theta band oscillations have a well-established role in working memory [47-49], and theta oscillations localized over the frontal lobe with frontoparietal extensions have repeatedly been suggested to reflect the transfer and integration of spatial information for the organization and maintenance of working and episodic memory [43-45]. In this theta function, the predominant strategy for the LH group can be simplified to preferential use of the frontal, particularly midline, activation pathways. Localized theta activity in this area extending into the lateral prefrontal regions are associated with error detection and general action monitoring [50]. The LH group's early trials present with general dispersion over the frontal lobe regions consolidating with task repetition, indicative of either decreased error or increased monitoring efficacy [44]. While present throughout, the later trials in all conditions had particularly concise connectivity over the medial frontal cortex, indicating a shift out of working memory and evidence of positive skill progression [51]. There was a slightly more anterior frontal focalization noticeable in the RH group, particularly in the later trials across conditions which may be indicative of ongoing goal-directed behavior and memory maintenance [48].

The visual nature of the motor task was expected to elicit parieto-occipital projections, a pathway with known visual-spatial function [52,53]. The parietal cortex has a particular role in sensory control of action coupled with activation of the occipital lobe in ongoing, sequential, visually guided behavior $[54,55]$. Both groups were expected to show this pattern, with a hypothesized greater bilateral involvement for LH and a comparatively lateralized connectivity pattern in $\mathrm{RH}$ [14]. Both groups did show evidence of attending to the task using visual input seen in occipital lobe connectivity with localized occipital lobe hubs across conditions for the initial trial bins. However, individuals in the LH group did not appear to use the parietal-occipital junction to the same extent as the RH group. The RH group had a commonly expressed hub at the temporal-parietal junction, an area well known for sensory integration [56], which may be related to the relationship between force output and cursor position in the RH group. Individuals in the RH group differentially exhibited classic activation patterns with known goal-directed spatial encoding functions [55]. This "classic activation" pattern may be a function of the majority of previous studies being exclusively conducted on right-handed individuals. While the LH group did initially exhibit occipital based hub across conditions, the group appeared to adopt a strategy based more on utilizing the ventral stream with some additional support of the dorsal stream showing differential projections of left lateral to left midline activity. This pattern is typically associated with transmitting visual information to the temporal lobe, often relaying highly processed descriptive information. This is indicative of a LH strategy that heavily relied on the visual information of the location or route of the target on the screen, to dictate the motor action [35]. With repeated task execution, the visual information became less influential, as seen by the decreased projections from the occipital lobe and a shift toward the midline. This likely reflects increased utilization of the dorsal stream, responsible for the decoding of spatial properties related to positional relations, motion direction, and speed of movement [52,53]. 
Based on the different patterns of theta connectivity, we suggest that LH and RH groups encoded the task differently. With different strategies and different uses of theta oscillations, hand dominant groups potentially optimize different aspects of the task, therein influencing skill acquisition tactics. The LH group presented with organized frontal midline activity. The RH group presented with some anterior frontal midline connectivity in the unimanual conditions that decreased in the dominant hand late trials, a pattern not seen in the non-dominant condition. In fact, in the late trials for both non-dominant and bimanual conditions, frontal theta activity appeared to increase, signifying ongoing error monitoring. These patterns are indicative of the state of the task, with the LH group expressing comparatively greater successful task encoding, while the RH group demonstrated a continued reliance on the working memory and visual input. The binned group averages of conditional graph metrics of theta connectivity patterns provide additional insight as to the neural strategy differences.

Assessing the LH and RH groups' raw connectivity patterns as a network, revealed differences in how the networks organized and responded to the task. As the degree or density of the coherent signal was modulated, either clustering coefficient or global efficiency would generally mirror. For LH dominant individuals, increased degree would yield an increase in the clustering coefficient. This nearly unanimous pattern suggests that an increase in signal density was used to increase local stability, perhaps yielding redundant or repetitive encoded messages. The increased global efficiency paired with decreased degree further supports this idea; a stable communication pattern fosters the emergence of a more efficient global integration of information. The slow oscillation of theta allows for the long-range integration of information, and it appears that integration was possible regardless of the condition. The activation networks of right-hand dominant individuals reflected the properties of different parameters. Generally, an increase in degree was followed by an increase in global efficiency and a decrease in clustering coefficient, the inverse pattern was also seen. The functionality of these patterns suggests the priority of global integration, perhaps associated with a reliance on propagating visual information.

Based on the quantification of network properties we can further speculate that LH individuals are more readily able to adapt and acquire the motor plan needed to successfully complete and encode the unimanual non-dominant motor task. The diminished degree and clustering coefficient paired with increased global efficiency between the final dominant and initial non-dominant and final non-dominant conditions support this successful transfer of motor strategy. In contrast, RH individuals had a less consistent modulation of the network metrics, global efficiency was increased in the dominant and bimanual conditions transitioning from initial to final trials but decreased between the initial to final trials in the non-dominant condition. While the clustering coefficient decreased between final dominant and non-dominant trial/conditions. Degree also behaved in a sporadic fashion, taken together the network behavior in the RH group alludes to an inconsistent strategy adopted by the RH individuals, with little progress maintained across conditions.

While both LH and RH groups showed a preference for distinct visual pathway networks, those were not the sole networks used. Taken together, the differential connectivity patterns and network metrics reflect a cognitively demanding task with enduring error monitoring, memory maintenance, and global and local integration. The rate of skill acquisition appears to be different between the two groups with the RH group unable to readily adapt to the use of their non-dominant hand as dexterously as their LH group counterpart. The different theta activation patterns suggest alternate uses of the slow oscillation, which is supported by the network metrics that were applied to the raw connectivity patterns that emerged across trials and conditions per group. While showing a stable strategy that relied on visual input integration, the RH group had less stable network parameters, seeming to rely on signal density and global integration to provide task stability. Alternately, the LH group had a more established progression relying on an increased clustering coefficient to gain stability followed by signal consolidation prior to global integration, seen in the inverse relationship of degree and global efficiency. 
These data suggest that $\mathrm{LH}$ and $\mathrm{RH}$ groups are using different optimization principles as well as performance strategies. These findings provide evidence that hand dominance influences strategies used to encode visual information necessary for hand motor output. Individuals in the LH group utilized a right lateral pathway, a strategy that mediated the organization of working memory, incorporating multiple components of information and memory maintenance. Individuals in the $\mathrm{RH}$ group relied on visual information to develop a strategy fit to the task. It follows that RH individuals, being in the majority population, can readily rely on a strategy based on integrating visual information to acquire a motor skill. Alternately, LH individuals, being in the population minority [57], may have found that encoded visual motor transformations are less reliable, which necessitates additional processing of visual feedback and more vigilant error monitoring when acquiring a motor skill. Practically, this is (potentially) reflective of most motor tasks being demonstrated by or designed for a right-hand dominant individual.

\section{Conclusions}

In nature, left-right asymmetry presents the rule rather than the exception, the universe itself has been found to exert a left-handed bias on particles [58,59]. Many motor control studies have examined functional regions of the brain in motor tasks, however, few have included left-hand dominant individuals. While the study does shed some light on how the two populations differ, it does more to highlight the unknown and validates the inclusion of this population in further studies of motor control and learning.

LH and RH dominant groups did present with overt strategic differences seen in the functional connectivity patterns of neural activity in the theta band. RH does appear to rely on a strategy that enforces local stability, seen in the increased clustering coefficient for all final trials and both bands. This strategy appears to be adopted with practice and was not transferable across conditions. The LH group appears to have greater success in skill transfer, showing a progressive increase in the clustering coefficient that continues after the shift from the dominant to the non-dominant hand condition with the final condition having a marked minimum in the theta band. This was interpreted as an expression of the successful acquisition of the skill, no longer requiring ongoing error monitoring to the same extent, or requiring the stability afforded by local interconnectedness. While changes were detectable and significant the two groups did not convincingly appear to have developed a fully stable motor pattern for the task. An extended study that incorporated multiple-day task execution would likely produce a more robust depiction of how hand-dominance influences the sensorimotor learning process.

Author Contributions: Conceptualization, J.M., N.P.M., S.C., E.E. and J.C.M.; data curation, J.M.; formal analysis, J.M., S.A. and J.C.M.; investigation, J.M.; methodology, J.M., S.A. and J.C.M.; project administration, J.M.; resources, N.P.M., S.C. and J.C.M.; software, J.M. and J.C.M.; supervision, N.P.M., S.A., S.C. and E.E.; validation, J.M. and N.P.M.; visualization, J.M., N.P.M., E.E. and J.C.M.; writing—original draft, J.M.; writing—review \& editing, J.M., S.A., S.C. and E.E. All authors have read and agreed to the published version of the manuscript.

Funding: This research received no external funding.

Institutional Review Board Statement: The study was conducted according to the guidelines of the Declaration of Helsinki, and approved by the Institutional Review Board of East Carolina University (UMCIRB 17-002599 01/19/2018).

Informed Consent Statement: Informed consent was obtained from all subjects involved in the study.

Data Availability Statement: The data presented in this study are available upon request from the corresponding author.

Acknowledgments: Authors would like to acknowledge the interns of the SMIL from 2014-2020 for their efforts and tenacity.

Conflicts of Interest: The authors declare no conflict of interest. 
Appendix A

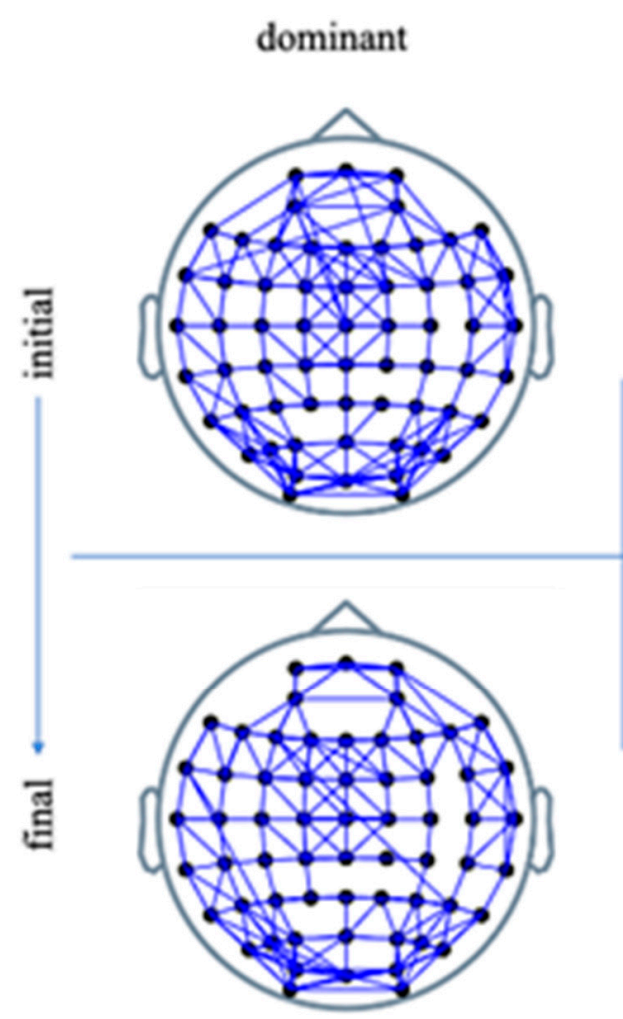

nondominant

bimanual
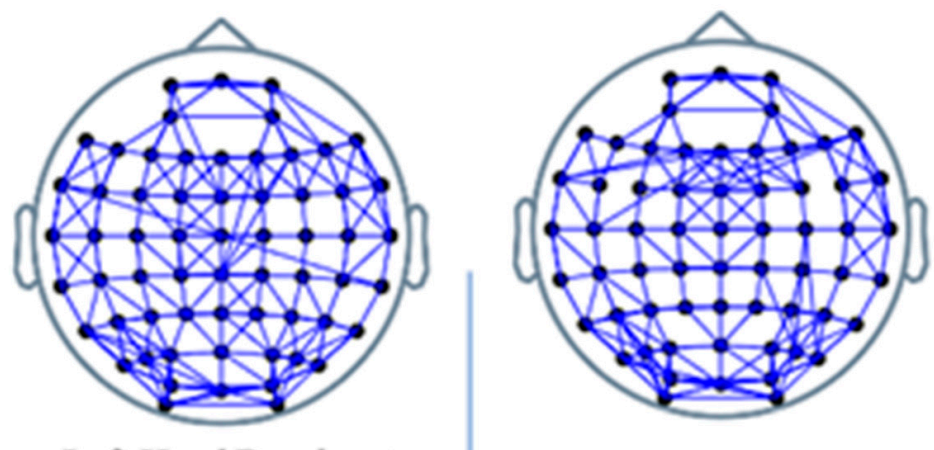

- Left Hand Dominant
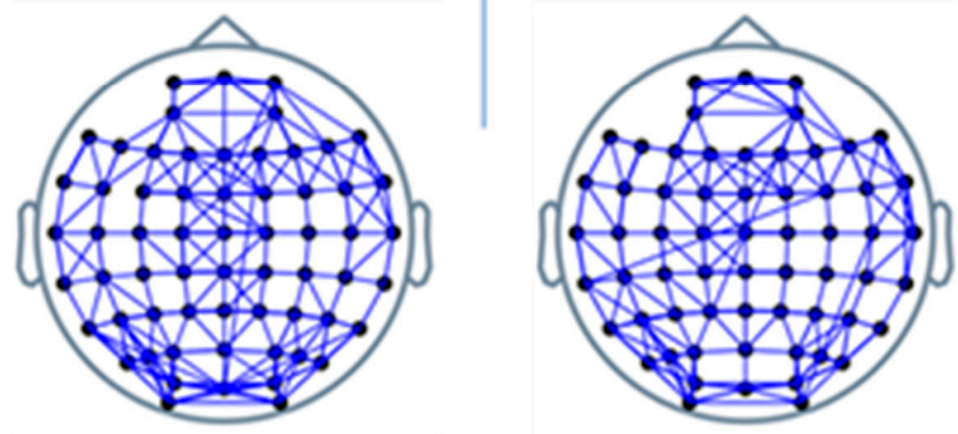

dominant

nondominant

bimanual
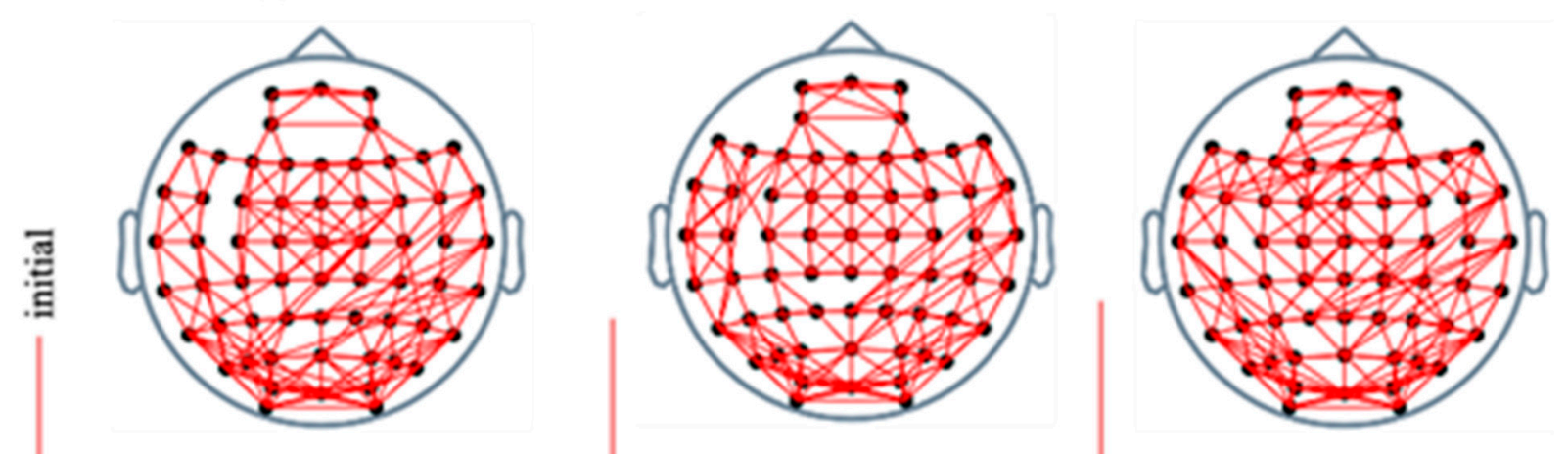

Right Hand Dominant-
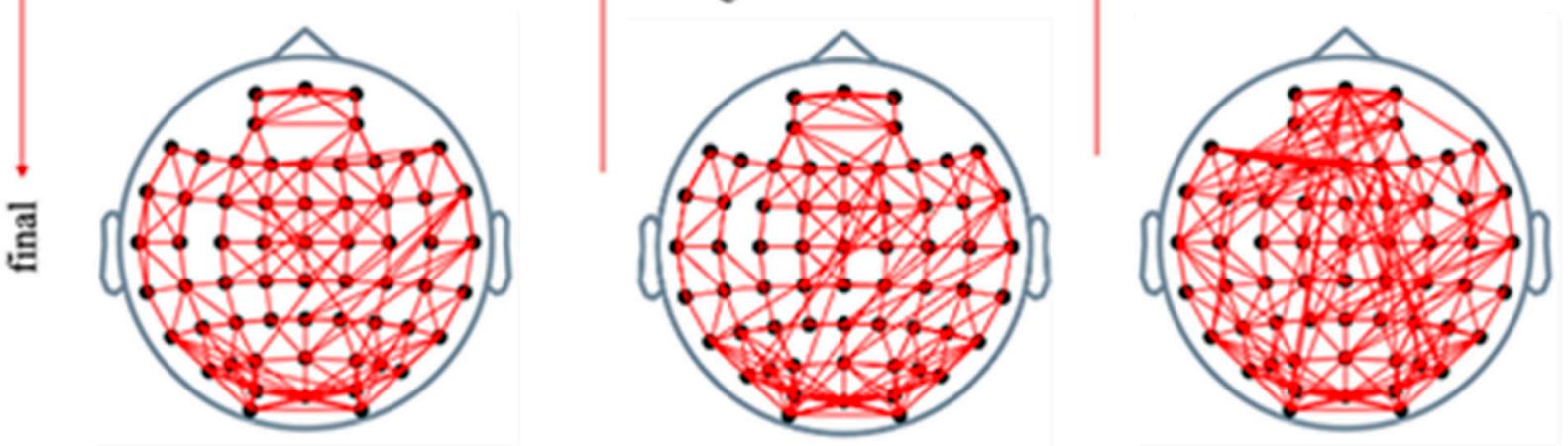

Figure A1. Left-hand dominant (blue) and right-hand dominant (red) significant neural activation patterns in the initial trial bins on the top row and final on the bottom with the column progressing from left to right with the conditions: unimanual dominant, non-dominant, and bimanual condition from left to right. 


\section{References}

1. Sporns, O.; Chialvo, D.R.; Kaiser, M.; Hilgetag, C.C. Organization, development and function of complex brain networks. Trends Cogn. Sci. 2004, 8, 418-425. [CrossRef]

2. Hagmann, P.; Cammoun, L.; Gigandet, X.; Meuli, R.; Honey, C.J.; Wedeen, V.J.; Sporns, O. Mapping the structural core of human cerebral cortex. PLoS Biol. 2008, 6, e159. [CrossRef]

3. Wolpert, D.M.; Ghahramani, Z.; Jordan, M.I. An internal model for sensorimotor integration. Science 1995, $269,1880-1882$. [CrossRef]

4. Miall, R.C.; Wolpert, D.M. Forward models for physiological motor control. Neural Netw. 1996, 9, 1265-1279. [CrossRef]

5. Wolpert, D.M. Computational approaches to motor control. Trends Cogn. Sci. 1997, 1, 209-216. [CrossRef]

6. Wolpert, D.M.; Ghahramani, Z. Computational principles of movement neuroscience. Nat. Neurosci. 2000, 3, 1212-1217. [CrossRef] [PubMed]

7. Vingerhoets, G.; Acke, F.; Alderweireldt, A.S.; Nys, J.; Vandemaele, P.; Achten, E. Cerebral lateralization of praxis in right-and left-handedness: Same pattern, different strength. Hum. Brain Mapp. 2012, 33, 763-777. [CrossRef] [PubMed]

8. Schmitz, J.; Metz, G.A.; Güntürkün, O.; Ocklenburg, S. Beyond the genome-Towards an epigenetic understanding of handedness ontogenesis. Prog. Neurobiol. 2017, 159, 69-89. [CrossRef]

9. Guadalupe, T.; Willems, R.M.; Zwiers, M.P.; Arias Vasquez, A.; Hoogman, M.; Hagoort, P.; Fernandez, G.; Buitelaar, J.; Franke, B. Differences in cerebral cortical anatomy of left-and right-handers. Front. Psychol. 2014, 5, 261. [CrossRef]

10. Przybyla, A.; Good, D.C.; Sainburg, R.L. Dynamic dominance varies with handedness: Reduced interlimb asymmetries in left-handers. Exp. Brain Res. 2012, 216, 419-431. [CrossRef]

11. Walker, E.H.; Perreault, E.J. Arm dominance affects feedforward strategy more than feedback sensitivity during a postural task. Exp. Brain Res. 2015, 233, 2001-2011. [CrossRef]

12. Knecht, S.; Dräger, B.; Deppe, M.; Bobe, L.; Lohmann, H.; Flöel, A.; Ringelstein, E.B.; Henningsen, H. Handedness and hemispheric language dominance in healthy humans. Brain 2000, 123, 2512-2518. [CrossRef] [PubMed]

13. Zietsch, B.P.; Hansen, J.L.; Hansell, N.K.; Geffen, G.M.; Martin, N.G.; Wright, M.J. Common and specific genetic influences on EEG power bands delta, theta, alpha, and beta. Biol. Psychol. 2007, 75, 154-164. [CrossRef]

14. Kelly, R.; Mizelle, J.; Wheaton, L.A. Distinctive laterality of neural networks supporting action understanding in left-and right-handed individuals: An EEG coherence study. Neuropsychologia 2015, 75, 20-29. [CrossRef] [PubMed]

15. Bailey, L.M.; McMillan, L.E.; Newman, A.J. A sinister subject: Quantifying handedness-based recruitment biases in current neuroimaging research. Eur. J. Neurosci. 2020, 51, 1642-1656. [CrossRef] [PubMed]

16. Petersen, P.; Petrick, M.; Connor, H.; Conklin, D. Grip strength and hand dominance: Challenging the 10\% rule. Am. J. Occup. Ther. 1989, 43, 444-447. [CrossRef] [PubMed]

17. Adamovich, S.V.; Fluet, G.G.; Tunik, E.; Merians, A.S. Sensorimotor training in virtual reality: A review. NeuroRehabilitation 2009, 25, 29-44. [CrossRef]

18. Wise, S.P.; Moody, S.L.; Blomstrom, K.J.; Mitz, A.R. Changes in motor cortical activity during visuomotor adaptation. Exp. Brain Res. 1998, 121, 285-299. [CrossRef]

19. Hadipour-Niktarash, A.; Lee, C.K.; Desmond, J.E.; Shadmehr, R. Impairment of retention but not acquisition of a visuomotor skill through time-dependent disruption of primary motor cortex. J. Neurosci. 2007, 27, 13413-13419. [CrossRef]

20. Muellbacher, W.; Ziemann, U.; Boroojerdi, B.; Cohen, L.; Hallett, M. Role of the human motor cortex in rapid motor learning. Exp. Brain Res. 2001, 136, 431-438. [CrossRef]

21. Da Silva, F.L. Neural mechanisms underlying brain waves: From neural membranes to networks. Electroencephalogr. Clin. Neurophysiol. 1991, 79, 81-93. [CrossRef]

22. Harmony, T. The functional significance of delta oscillations in cognitive processing. Front. Integr. Neurosci. 2013, 7, 83. [CrossRef]

23. Oldfield, R.C. The assessment and analysis of handedness: The Edinburgh inventory. Neuropsychologia 1971, 9, 97-113. [CrossRef]

24. Amunts, K.; Armstrong, E.; Malikovic, A.; Hömke, L.; Mohlberg, H.; Schleicher, A.; Zilles, K. Gender-specific left-right asymmetries in human visual cortex. J. Neurosci. 2007, 27, 1356-1364. [CrossRef] [PubMed]

25. Ocklenburg, S.; Gunturkun, O. The Lateralized Brain: The Neuroscience and Evolution of Hemispheric Asymmetries; Academic Press: Cambridge, MA, USA, 2017.

26. Proverbio, A.M.; Brignone, V.; Matarazzo, S.; Del Zotto, M.; Zani, A. Gender differences in hemispheric asymmetry for face processing. BMC Neurosci. 2006, 7, 1-10. [CrossRef] [PubMed]

27. Ghahramani, Z.; Wolpert, D.M. Modular decomposition in visuomotor learning. Nature 1997, 386, 392. [CrossRef] [PubMed]

28. Wolpert, D.M.; Flanagan, J.R. Motor learning. Curr. Biol. 2010, 20, R467-R472. [CrossRef]

29. Sailer, J.; Scharitzer, M.; Peloschek, P.; Giurea, A.; Imhof, H.; Grampp, S. Quantification of axial alignment of the lower extremity on conventional and digital total leg radiographs. Eur. Radiol. 2005, 15, 170-173. [CrossRef] [PubMed]

30. Braun, D.A.; Aertsen, A.; Wolpert, D.M.; Mehring, C. Motor task variation induces structural learning. Curr. Biol. 2009, 19, 352-357. [CrossRef]

31. Sailer, U.; Flanagan, J.R.; Johansson, R.S. Eye-hand coordination during learning of a novel visuomotor task. J. Neurosci. 2005, 25, 8833-8842. [CrossRef]

32. Delorme, A.; Makeig, S. EEGLAB: An open source toolbox for analysis of single-trial EEG dynamics including independent component analysis. J. Neurosci. Methods 2004, 134, 9-21. [CrossRef] 
33. Mullen, T.; Kothe, C.; Chi, Y.M.; Ojeda, A.; Kerth, T.; Makeig, S.; Cauwenberghs, G.; Jung, T.P. Real-time modeling and 3D visualization of source dynamics and connectivity using wearable EEG. In Proceedings of the Engineering in Medicine and Biology Society (EMBC), 2013 35th Annual International Conference of the IEEE, Osaka, Japan, 3-7 July 2013; IEEE: Piscataway, NJ, USA, 2013.

34. Kayser, J.; Tenke, C.E. On the benefits of using surface Laplacian (current source density) methodology in electrophysiology. Int. J. Psychophysiol. Off. J. Int. Organ. Psychophysiol. 2015, 97, 171. [CrossRef] [PubMed]

35. Nolte, G.; Bai, O.; Wheaton, L.; Mari, Z.; Vorbach, S.; Hallett, M. Identifying true brain interaction from EEG data using the imaginary part of coherency. Clin. Neurophysiol. 2004, 115, 2292-2307. [CrossRef]

36. Ewald, A.; Aristei, S.; Nolte, G.; Rahman, R.A. Brain oscillations and functional connectivity during overt language production. Front. Psychol. 2012, 3, 166. [CrossRef] [PubMed]

37. Shamas, M.; Wendling, F.; El Falou, W.; Hassan, M. EEGNET: A novel tool for processing and mapping EEG functional networks. In Proceedings of the 2015 7th International IEEE/EMBS Conference on Neural Engineering (NER), Montpellier, France, 22-24 April 2015; IEEE: Piscataway, NJ, USA, 2015.

38. Rubinov, M.; Sporns, O. Complex network measures of brain connectivity: Uses and interpretations. Neuroimage 2010, 52, 1059-1069. [CrossRef] [PubMed]

39. Mijalkov, M.; Kakaei, E.; Pereira, J.B.; Westman, E.; Volpe, G.; Alzheimer's Disease Neuroimaging Initiative. BRAPH: A graph theory software for the analysis of brain connectivity. PLoS ONE 2017, 12. [CrossRef]

40. Bullmore, E.; Sporns, O. The economy of brain network organization. Nat. Rev. Neurosci. 2012, 13, 336. [CrossRef]

41. Finotellia, P.; Dulioa, P. Graph. Theoretical Analysis of the Brain. An. Overview. Sci. Ric. 2015, 9, 89-96.

42. Maris, E.; Oostenveld, R. Nonparametric statistical testing of EEG-and MEG-data. J. Neurosci. Methods 2007, 164, 177-190. [CrossRef]

43. Cavanagh, J.F.; Frank, M.J. Frontal theta as a mechanism for cognitive control. Trends Cogn. Sci. 2014, 18, 414-421. [CrossRef]

44. Caplan, J.B.; Madsen, J.R.; Schulze-Bonhage, A.; Aschenbrenner-Scheibe, R.; Newman, E.L.; Kahana, M.J. Human $\theta$ oscillations related to sensorimotor integration and spatial learning. J. Neurosci. 2003, 23, 4726-4736. [CrossRef]

45. Lisman, J.; Buzsáki, G. A neural coding scheme formed by the combined function of gamma and theta oscillations. Schizophr. Bull. 2008, 34, 974-980. [CrossRef] [PubMed]

46. Mizelle, J.C.; Forrester, L.; Hallett, M.; Wheaton, L.A. Theta frequency band activity and attentional mechanisms in visual and proprioceptive demand. Exp. Brain Res. 2010, 204, 189-197. [CrossRef]

47. Klimesch, W. EEG alpha and theta oscillations reflect cognitive and memory performance: A review and analysis. Brain Res. Rev. 1999, 29, 169-195. [CrossRef]

48. Raghavachari, S.; Lisman, J.E.; Tully, M.; Madsen, J.R.; Bromfield, E.B.; Kahana, M.J. Theta oscillations in human cortex during a working-memory task: Evidence for local generators. J. Neurophysiol. 2006, 95, 1630-1638. [CrossRef] [PubMed]

49. Raghavachari, S.; Kahana, M.J.; Rizzuto, D.S.; Caplan, J.B.; Kirschen, M.P.; Bourgeois, B.; Madsen, J.R.; Lisman, J.E. Gating of human theta oscillations by a working memory task. J. Neurosci. 2001, 21, 3175-3183. [CrossRef]

50. Devinsky, O.; Morrell, M.J.; Vogt, B.A. Contributions of anterior cingulate cortex to behaviour. Brain 1995, 118, 279-306. [CrossRef]

51. Klimesch, W.; Schimke, H.; Schwaiger, J. Episodic and semantic memory: An analysis in the EEG theta and alpha band. Electroencephalogr. Clin. Neurophysiol. 1994, 91, 428-441. [CrossRef]

52. Goodale, M.A.; Milner, A.D. Separate visual pathways for perception and action. Trends Neurosci. 1992. [CrossRef]

53. Willingham, D.B. A neuropsychological theory of motor skill learning. Psychol. Rev. 1998, 105, 558. [CrossRef]

54. Roux, F.; Uhlhaas, P.J. Working memory and neural oscillations: Alpha-gamma versus theta-gamma codes for distinct WM information? Trends Cogn. Sci. 2014, 18, 16-25. [CrossRef] [PubMed]

55. Blanke, O.; Arzy, S. The out-of-body experience: Disturbed self-processing at the temporo-parietal junction. Neuroscientist 2005, 11, 16-24. [CrossRef] [PubMed]

56. Hutchinson, J.B.; Uncapher, M.R.; Wagner, A.D. Posterior parietal cortex and episodic retrieval: Convergent and divergent effects of attention and memory. Learn. Mem. 2009, 16, 343-356. [CrossRef] [PubMed]

57. Papadatou-Pastou, M.; Ntolka, E.; Schmitz, J.; Martin, M.; Munafò, M.R.; Ocklenburg, S.; Paracchini, S. Human handedness: A meta-analysis. Psychol. Bull. 2020. [CrossRef]

58. Good, C.D.; Johnsrude, I.; Ashburner, J.; Henson, R.N.; Friston, K.J.; Frackowiak, R.S. Cerebral asymmetry and the effects of sex and handedness on brain structure: A voxel-based morphometric analysis of 465 normal adult human brains. Neuroimage 2001, $14,685-700$.

59. Cowan, G.A.; Craik, D.C.; Needham, M. RapidSim: An application for the fast simulation of heavy-quark hadron decays. Comput. Phys. Commun. 2017, 214, 239-246. [CrossRef] 